$63^{\text {ème }}$ Congrès de la SFCO, 02019 (2015)

DOI:10.1051/sfco/20156302019

(C) Owned by the authors, published by EDP Sciences, 2015

\title{
COMMUNICATION
}

\section{La pression artérielle de repos : un facteur prédictif de la sévérité de la douleur postopératoire aigüe en chirurgie orale}

\author{
Deschaumes $\mathrm{C}^{* * * *}$, Devoize $\mathrm{L}^{* * * *}$, Sudrat $\mathrm{Y}^{* * * *}$, Baudet-Pommel $\mathrm{M}^{* * * *}$, Dualé $\mathrm{C}^{*, * * *}$, \\ Dallel $\mathrm{R}^{* * * *}$ \\ * Clermont Université, Université d'Auvergne, Neuro-Dol, BP. 10448, 63000 Clermont-Ferrand \& Inserm \\ U1107, 63001 Clermont-Ferrand \\ ** CHU Clermont-Ferrand, Service d'Odontologie, 63003 Clermont-Ferrand \\ *** CHU Clermont-Ferrand, Inserm, CIC 50, 63003 Clermont-Ferrand
}

La douleur aigue post-opératoire reste encore aujourd'hui un enjeu clinique important car variable d'un individu à un autre et associée à un risque élevé de développer une douleur chronique (Dworkin 1997). De nombreuses études tant expérimentales que cliniques montrent le caractère multifactoriel de la douleur post-opératoire. Outre les paramètres chirurgicaux, l’intensité de la douleur post-opératoire perçue varie en fonction de paramètres individuels. Des interactions fonctionnelles ont été mises en évidence entre le système cardiovasculaire et les systèmes de régulation de la douleur (Ghione 1996, Bruehl 2004).

L’objectif de cette étude clinique prospective est d’identifier les facteurs prédictifs de la sévérité de la douleur aigue après avulsion dentaire. Elle vise notamment à évaluer la relation de la pression artérielle de repos, decertains paramètres démographiques, anatomiques (liés aux dents avulsées) et opératoires, avec l'intensité de la douleur.

Matériel et méthode

Dans cette étude prospective (CPP Sud Est -AU567), 293 patients devant bénéficier l'avulsion d'une ou plusieurs dents sous anesthésie locale en ambulatoire au CHRU de Clermont Ferrand ont été inclus de septembre 2005 à mars 2010.

La douleur post-opératoire a été évaluée à l'aide un score composite de douleur construit avec, pour part, l'intensité de la douleur spontanée les 6 premières heures et pour part, la consommation antalgique durant les 3 premiers jours post-opératoires

Le critère principal de l'étude est la pression artérielle de repos.

Les critères secondaires sont d'ordre démographiques (âge, sexe, antécédents médicaux et chirurgicaux, facteurs psychologiques, antécédents de douleurs orales), chirurgicaux (sites d’intervention, nombre et type de dents avulsées), anatomiques (intégrité dentaire, degré d’inclusion) et hémodynamiques (pression artérielle de repos, rythme cardiaque).

This is an Open Access article distributed under the terms of the Creative Commons Attribution License 4.0, which permits unrestricted use, distribution, and reproduction in any medium, provided the original work is properly cited. 


\section{Résultats}

L'analyse univariée montre que l'intensité de douleur post-opératoire varie selon certains paramètres.

Les facteurs prédisposants à une douleur importantes sont la durée d'intervention, la multiplicité des sites d'avulsion (maxillaire et mandibule), le nombre de dents concernées, l'avulsion d'une $3^{\text {ème }}$ molaire et l'inclusion des dents avulsées. Par contre, certains paramètres peuvent être considérés comme protecteurs vis à vis de la douleur postopératoire : ce sont l'âge, un antécédent d'hypertension artérielle, une pression artérielle de repos pré opératoire élevée et l'existence d'un antécédent de chirurgie orale).

De ces paramètres, seuls certains sont considérés par l'analyse multivariée comme prédictifs de l'intensité de la douleur postopératoire. Ce sont des paramètres individuels (âge et pression artérielle moyenne de repos préopératoire), des paramètres chirurgicaux et anatomiques (le caractère bimaxillaire de la chirurgie et le degré d'inclusion des dents).

Conclusions

Cette étude montre qu'une pression artérielle de repos préopératoire élevée est un facteur protecteur vis à vis de la douleur post-opératoire. Sa mesure avant l'intervention pourrait aider à identifier les patients à risque de développer une douleur post-chirurgicale sévère.

Nom et adresse du conférencier

\section{Christophe DESCHAUMES}

CHU - Service d'Odontologie

1 place Raymond et Lucie Aubrac

63003 Clermont-Ferrand (France)

christophe.deschaumes@udamail.fr 